Cal cul at $i$ on of sel $f$-diffusi on coef $f i$ ci ent s of the [BM M [ TFSA] /wat er syst em by nol ecul ar dynami cs si mul at $i$ on

\begin{tabular}{|l|l|}
\hline 著者 & $\begin{array}{l}\text { H gashi Hi denor i , Kum t a M ki ko, Set o } \\
\text { Takaf um, Ot ani Yoshi o }\end{array}$ \\
\hline $\begin{array}{l}\text { j our nal or } \\
\text { publ i cat i on titl e }\end{array}$ & Mbl ecul ar Si mul at i on \\
\hline vol une & 43 \\
\hline number & 17 \\
\hline page range & $1430-1435$ \\
\hline year & $2017-11-22$ \\
\hline URL & ht t p: //hdl . handl e. net $/ 2297 / 48645$ \\
\hline
\end{tabular}




\title{
Calculation of Self-diffusion Coefficients of the [BMIM][TFSA]/Water System by Molecular Dynamics Simulation
}

\author{
Hidenori HIGASHI*, Mikio KUMITA, Takafumi SETO and Yoshio OTANI \\ Faculty of Natural Systems, Institute of Science and Engineering, Kanazawa University, Kakuma-machi, \\ Kanazawa, 920-1192, Japan \\ * Correspondence concerning this article should be addressed to H. Higashi (E-mail: \\ hhigashi@mail.kanazawa-u.ac.jp).
}


We performed a molecular dynamics simulation to calculate the self-diffusion coefficients of 1-Butyl-3-methylimidazolium bis(trifluoromethanesulfonyl)imide and water in a water-ionic liquid mixture. We then compared the simulated self-diffusion coefficients of cation, anion, and water molecules with experimental data and with simulated data from the literature. Although the simulation overestimated the self-diffusion coefficients of ions, the simulated results qualitatively reproduced the enhancement of the self-diffusion coefficients of water as the water molar fraction increased. We also calculated the radial distribution functions to investigate the solution structure, i.e., the clustering of water molecules. The clustering of water in ionic liquid was found to play an important role in the enhancement of the diffusion of water molecules in the ionic liquid.

Keywords: molecular dynamics, self-diffusion coefficient, ionic liquid, water, radial distribution function 


\section{INTRODUCTION}

Ionic liquids have very low vapor pressure and are expected to be adopted as a new class of separation and reaction solvents in place of harmful organic solvents. They have also been applied to energy storage devices in recent years. A growing body of data is being collected on the fundamental properties of ionic liquids, such as their phase equilibrium, diffusion coefficients, and viscosity. Some ionic liquids have high affinity with water and are often used as water-ionic liquid solvents. 1-Butyl-3- methylimidazolium bis(trifluoromethane sulfonyl)imide ([BMIM][TFSA]) is a high viscosity hydrophobic ionic liquid and it is already serves as a useful electrolyte solution because its properties are well-investigated.

Tokuda et al. [1-3] reported the transport properties of various ionic liquids and proposed equations to predict them. Filippov et al. $[4,5]$ reported the self-diffusion of a phosphonium bis(mandelato)borate ionic liquid and mixtures of imidazolium bis(mandelato)borate ionic liquids with polyethylene glycol by nuclear magnetic resonance (NMR). Kruk et al. [6] measured the self-diffusion coefficients of ionic liquids by the field cycling nuclear magnetic resonance (FC NMR) relaxation method and reported that the diffusion coefficients obtained were in good agreement with those measured by pulsed field gradient (PFG) NMR. Rollet et al. [7] measured the diffusion coefficient of [BMIM][TFSA] (or TFSI according to their nomenclature) by NMR with water added to the solution. They found that the self-diffusion coefficients of water rapidly increased with a rising water concentration, even when the water molar fraction was as low as 0.30 . The enhancement of diffusion coefficients of water are much larger than the estimated values by the Stokes-Einstein relation. They only showed the snap shots of molecular dynamics (MD) simulation of water-ionic liquid mixture. Judging from the results of the MD simulation, they attributed the significant enhancement of the self-diffusion coefficients to the existence of a 'water pool' in the ionic liquid. They took no steps, however, in their MD simulations, to calculate the self-diffusion coefficients or investigate the enhancement of self-diffusion of water in ionic liquid. Menjoge et al. [8] applied a novel PFG NMR option to study the diffusion behavior of cation, anion, and water molecules in two 1-ethyl-3-methylimidazolium-based ionic liquids.

They discussed how the addition of water influenced the diffusion behavior of the cation and anion by concerning the 
presence of polar and nonpolar domains in the ionic liquids. They surmised that the subsequent changes in the diffusion behavior in their experiments stemmed from a partial screening of the electrostatic interaction between the cation and anion in the polar domains by water. They found that water-ionic liquid mixtures behaved uniquely, leading to anomalous enhancements of the water diffusivities as the water concentration rose. Their results underlined the need to analyze the diffusion mechanism of the water-ionic liquid system from the viewpoint of molecular theory.

MD simulation is a powerful tool for estimating the transport properties of fluid. Several reports have described the calculation of diffusion coefficients in ionic liquids by MD simulation (Kowsari et al. [9, 10], Jiang et al. [11], Chen et al. [12] and Matsumiya et al. [13]). Tsuzuki et al. [14] conducted MD simulations to calculate the self-diffusion coefficients of a series of ionic liquids (1-alkyl-3-methylimidazolium, $N$-butyl- $N, N, N-\quad$ trimethyl-ammonium and $\mathrm{N}$-butyl- $\mathrm{N}$-methylpyrrolidinium cations combined with a $\left(\mathrm{CF}_{3} \mathrm{SO}_{2}\right)_{2} \mathrm{~N}-$ anion ([mmim][TFSA], [emim][TFSA], [bmim][TFSA], etc.) and a 1-butyl-3-methylimidazolium combined with various anions). Their simulations, however, underestimated the experimental data. Two approaches have since been proposed to solve this problem, namely, to use polarizable models or to scale the partial charges leading to ionic charges different from unity. Köddermann et al. [15] modified the Lennard-Jones parameters for united-atom model of imidazolium-based ionic liquids to represent the transport properties. Zhong et al. [16] also proposed the parameters for united-atom model. Niazi et al. [17] applied the latter approach when performing an MD simulation of the diffusion behavior of the cation and anion in imidazolium salt-based ionic liquids with wide-ranging molar fractions of water added to the system. They reported that the diffusion rates of the cation and anion in imidazolium-based ionic liquids rapidly increased as a function of the water concentration, especially at high water concentrations over $70 \mathrm{~mol} \%$, in spite of a significant "crossover" occurring at around that mole fraction. There has been no detailed discussion, however, on the solution structure or diffusivity of ions and water molecules at lower water concentration.

In the present work we performed an MD simulation of a [BMIM][TFSA]/water system and calculated the 
self-diffusion coefficients of cation, anion, and water molecules, particularly at low water molar fractions $(<0.30)$. We applied the rigid model for water in the [BMIM][TFSA]/water system and employed flexible all-atom models for the cation and anion. Simulated self-diffusion coefficients of cation, anion, and water molecules were compared with simulated data from Tsuzuki et al. [14] and with experimental data from Rollet et al. [7] and Tokuda et al. [1]. We then examined how well the molecular models described the anomalous diffusion behavior of water in ionic liquid. Finally, we calculated the radial distribution functions for ions and water to investigate the solution structure of the mixture.

\section{Molecular Simulation}

\subsection{Potentials}

A formula obtained by adding the interaction of charges to the Lennard-Jones (12-6) type of potential was assumed to represent the non-bonding potential energy interacting between the atoms. The Ewald sum method [18] was adopted to calculate the electrical charge interaction. The cutoff length of the non-bonding interaction was set to $15 \AA$. The SPC/E model (Berendsen et al. [19]) was applied for water by assuming the water molecule to be rigid. Lee [20] revealed the applicability of the model to calculate the self-diffusion coefficients of water. In the present study, an extension of the model to the mixture was investigated by simulating diffusion behavior of water in ionic liquid. The potential function of the ions was expressed by an all-atom model with the bonding interaction added. The potential parameters and electrical charges of each atom of the cation and anion were taken from Köddermann et al. [15]. All of the C-H bonds were treated as rigid using the SHAKE algorithm by Tsuzuki et al. [14], whereas all of the atoms were treated as flexible.

\subsection{Simulation conditions}

The

$$
\text { simulation }
$$$$
\text { was }
$$

performed

by

commercial

software 
anion, and water molecules were constructed in the system. The box size of the present simulation, about $32 \AA$, was much larger than the cut-off length of the Lennard-Jones potential $(9 \AA)$ and more than adequate to calculate the molecular behavior of the water-ionic liquid mixtures. The water concentration was adjusted by changing the ratio of ions and water molecules.

The Lorentz-Berthelot law was used to determine the parameters between unlike atoms. The time step for solving the equations of motion was set to $1 \mathrm{fs}$. After molecules placed randomly in a cubic cell were subjected to periodic boundary conditions, a pre-equilibration calculation was performed at low density and high temperature. The system was then mixed by $5.0 \times 10^{4}$ steps $(50 \mathrm{ps})$ of an equilibration $N V T$ calculation at high temperature. The system conditions were gradually adjusted to the simulated temperatures $(303,353$ and $453 \mathrm{~K}$ for the [BMIM][TFSA] system and $298 \mathrm{~K}$ for the [BMIM][TFSA]/water system) and atmospheric pressure by $5.0 \times 10^{5}$ steps (500 ps) of the equilibration NPT calculation. The temperature and pressure were controlled by a Nose-Hoover thermostat (Nose [21] and Hoover [22]) and barostat method (Berendsen et al. [23]). The production calculation was then performed for $1.2 \times 10^{7}$ steps (12 ns) and the data were saved every $10 \mathrm{ps}$.

\subsection{Determination of self-diffusion coefficients}

The self-diffusion coefficients were calculated from the slope of the mean square displacement (MSD) by the Einstein relationship:

$D_{i}=\lim _{t \rightarrow \infty} \frac{1}{6 t}\left\langle\left[\mathbf{r}_{i}(t)-\mathbf{r}_{i}(0)\right]^{2}\right\rangle$

where $D$ is the self-diffusion coefficient, $i$ is the component, $t$ is the time, and $\mathbf{r}$ is the position of the center of mass of the component. The calculated self-diffusion coefficients of cation and anion molecules for the [BMIM][TFSA] system were compared with the experimental values from Tokuda et al. [1] and with the simulated data from Köddermann et al. [15] and Zhong et al. [16]. 


\subsection{Radial distribution function}

The radial distribution function describes how density varies as a function of distance from a reference molecule and denotes the solution structure:

$g(r)=4 \pi r^{2} \rho(r) d r$

where $\rho$ is the number density and $r$ is the distance from the reference molecule. In this work, the radial distribution functions for the center of mass of each molecule were calculated from all trajectories of the simulation. The radial distribution functions between $\mathrm{H}_{2} \mathrm{O}$-cation, $\mathrm{H}_{2} \mathrm{O}$-anion, cation-anion, and $\mathrm{H}_{2} \mathrm{O}-\mathrm{H}_{2} \mathrm{O}$ were calculated to investigate the solution structure of the $[\mathrm{BMIM}][\mathrm{TFSA}] /$ water system.

\section{Results and Discussion}

\subsection{Self-diffusion coefficients in [BMIM][TFSA]}

The self-diffusion coefficients of cation and anion molecules for the [BMIM][TFSA] system at 303, 353 and $453 \mathrm{~K}$ were determined from the slope of the MSD by the Einstein relationship (Eq.1). Figure 1 shows the (a) $\log -\log$ and (b) normal plots of the calculated result of the MSD as a function of time. The results calculated by Ivanovskis et al. [24] are also shown in the figure. In our calculations, a linear relationship between the MSD and time was obtained over 200 ps as shown in Figure 1(b). For slopes longer than 2,000 ps, the calculation results were unreliable and unreproducible due to a lack of statistics. The slopes of the MSDs of the ions were therefore determined over the time interval from 500 to $1,000 \mathrm{ps}$. The time interval adopted in this work is comparable to that of Ivanovskis et al. [24]. On the other hand, de Andrade et al. [25] and Liu et al. [26] selected shorter time intervals to determine the self-diffusion coefficients of the ions. Sufficiently abundant simulation data were obtained in our time interval to statistically represent normal diffusion behavior as shown in Figure 1 (a), because the slope of log-log plots of MSD over 200 ps is almost unity. It is also indicated that the simulation run length is enough to calculate the diffusion coefficients. The simulated results in this work are the same order of the experimental 

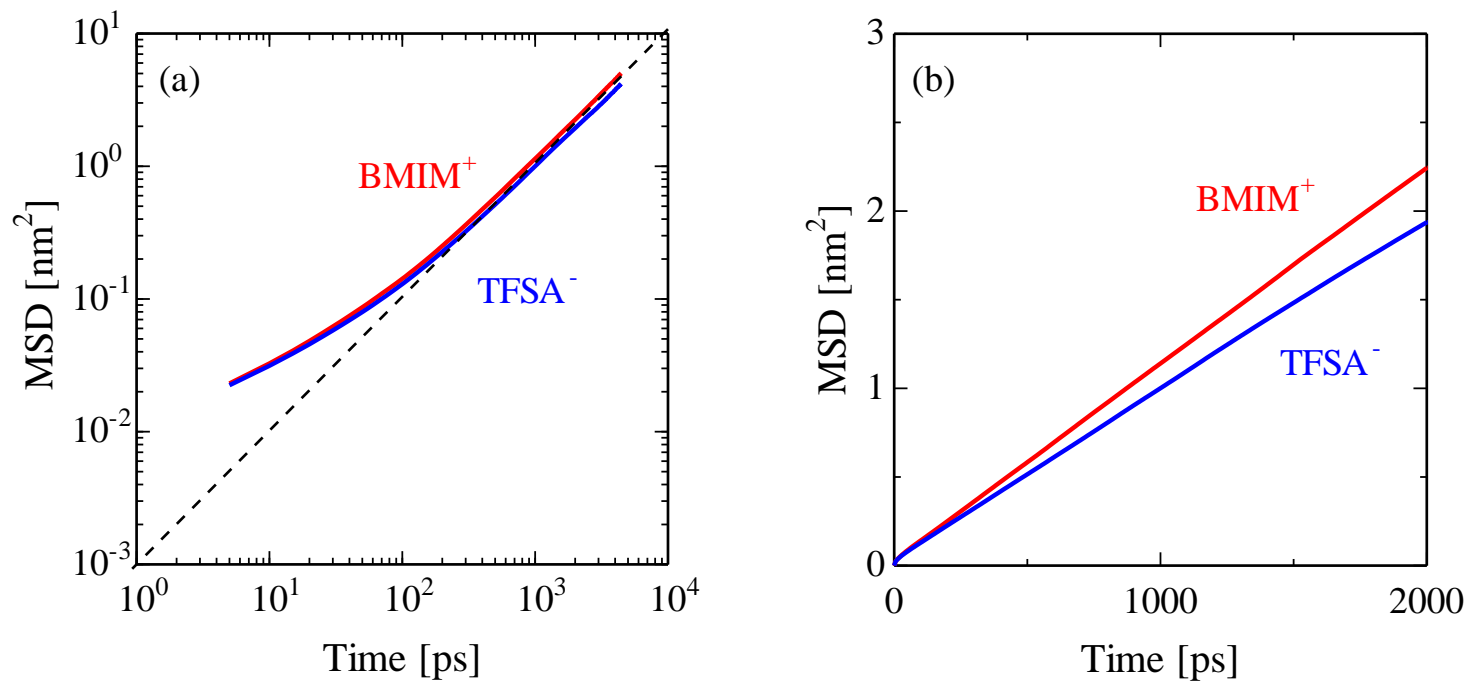

Figure 1 Mean square displacements of $\mathrm{BMIM}^{+}$and TFSA- molecules at $353 \mathrm{~K}$

The simulated density and self-diffusion coefficients are listed in Table 1. The densities shown in the table are averaged for all simulation times. The results agreed well with the experimental data of Tokuda et al. [1]. The self-diffusion coefficients of the ions calculated in Tsuzuki et al. [14] were notably lower than the self-diffusion coefficients determined experimentally. It would be suspected that this disagreement could be a fairly common problem in the application of the classical models and their parameters for ionic liquids. On the other hands, the self-diffusion coefficients of ions calculated in Köddermann et al. [15], Zhong et al. [16] and the present work are well represented the experimental data by the classical models with the modified Lennard-Jones parameters. As a first step to the simulation for ionic liquid and water mixture system, we have applied the classical water and ionic liquid models without further modification to discuss the anomalous diffusion behavior of water in ionic liquids. 
Table 1 Densities and self-diffusion coefficients of $\mathrm{BMIM}^{+}$and $\mathrm{TFSA}^{-}$calculated by molecular dynamics simulation

\begin{tabular}{|c|c|c|c|c|c|c|c|c|c|c|c|c|c|}
\hline \multicolumn{2}{|c|}{$T[\mathrm{~K}]$} & \multicolumn{5}{|c|}{303} & \multicolumn{4}{|c|}{353} & \multicolumn{3}{|c|}{453} \\
\hline & & \multicolumn{3}{|c|}{ Sim. } & & \multirow{2}{*}{$\begin{array}{c}\text { Exp. }{ }^{* 1} \\
\text { Tokuda } \\
\text { et al. } \\
{[1]}\end{array}$} & \multicolumn{3}{|c|}{ Sim. } & \multirow{2}{*}{$\begin{array}{c}\text { Exp. }{ }^{* 1} \\
\text { Tokuda } \\
\text { et al. } \\
{[1]}\end{array}$} & \multicolumn{2}{|c|}{ Sim. } & \multirow{2}{*}{$\begin{array}{c}\text { Exp. }{ }^{* 1} \\
\text { Tokuda } \\
\text { et al. } \\
{[1]}\end{array}$} \\
\hline & & $\begin{array}{l}\text { Tsuzuki } \\
\text { et al. }[14]\end{array}$ & $\begin{array}{c}\text { Ködderm } \\
\text { ann et al. } \\
{[15]}\end{array}$ & $\begin{array}{c}\text { Zhong } \\
\text { et al. } \\
{[16]}\end{array}$ & $\begin{array}{l}\text { This } \\
\text { work }\end{array}$ & & $\begin{array}{l}\text { Tsuzuki } \\
\text { et al. [14] }\end{array}$ & $\begin{array}{c}\text { Zhong } \\
\text { et al. } \\
{[16]}\end{array}$ & $\begin{array}{l}\text { This } \\
\text { work }\end{array}$ & & $\begin{array}{l}\text { Tsuzuki } \\
\text { et al. }[14]\end{array}$ & $\begin{array}{l}\text { This } \\
\text { work }\end{array}$ & \\
\hline \multicolumn{2}{|c|}{$\rho\left[\mathrm{g} / \mathrm{cm}^{3}\right]$} & $1.431^{* 2}$ & - & 1.364 & 1.39 & 1.435 & 1.414 & 1.329 & 1.32 & 1.388 & - & 1.21 & 1.294 \\
\hline$D \times 10^{10}$ & $\mathrm{BMIM}^{+}$ & - & 0.34 & - & 0.45 & 0.30 & 0.078 & 1.09 & 1.85 & 1.48 & 1.20 & 7.91 & 6.33 \\
\hline$\left[\mathrm{m}^{2} / \mathrm{s}\right]$ & TFSA $^{-}$ & - & 0.26 & - & 0.40 & 0.23 & 0.059 & 0.78 & 1.61 & 1.25 & 0.97 & 6.81 & 5.86 \\
\hline
\end{tabular}

*1 Experimental values were calculated by the equations proposed by Tokuda et al. [1].

*2 Simulated density by Tsuzuki et al. [14] was calculated at $298 \mathrm{~K}$.

\subsection{Self-diffusion coefficients of the [BMIM][TFSA]/water system}

Figure 2 shows the MSD of cation, anion, and water molecules as a function of time for the [BMIM][TFSA]/water system. A linear relationship between the MSD and time was also obtained over 200 ps as shown in Fig.2. For slopes longer than 2,000 ps, the calculation results were unreliable and unreproducible due to a lack of statistics for small amount of water molecules. The diffusion coefficients were determined by the slopes of the MSDs over the time interval from 500 to 1,000 ps.

The slopes of the MSDs of the cation and anion molecules are almost the same at all water molar fractions, suggesting that cation and anion molecules paired and moved together. The slopes of the MSDs of the water molecules, meanwhile, were much larger than those of the ions. We also found that the slopes of the MSDs of the cation, anion and water molecules gradually increased as the water molar fraction rose (see Fig. 2). In particular, the slope of the MSD of the water molecule was more significantly enhanced with increasing $x_{\mathrm{H} 2 \mathrm{O}}$ than the slopes of the MSDs of the cation and anion.

Table 2 and Figure 3 show the calculated self-diffusion coefficients of the cation, anion, and water molecules as a function of the water molar fraction in the [BMIM][TFSA]/water system at $298 \mathrm{~K}$. The experimental data by Rollet et al. [7] is also plotted in Fig. 3. While the calculated results of the self-diffusion coefficients of the cation and anion showed twice of 
the experimental values, the tendency of increase in the diffusion coefficients with molar fraction of water could be represented. As mentioned previously, the experimental self-diffusion coefficients of water (closed plots) exhibited strong (anomalous) dependence on the water molar fraction. The simulated results (open plots) qualitatively reproduced the small dependence of water molar fraction. Note that the calculation may contain some error at the minimum water molar fraction $\left(x_{\mathrm{H} 2 \mathrm{O}}=0.03\right)$ because the system contains only three water molecules. We can therefore conclude that the simulation qualitatively represents the effect of the water concentration on the diffusivity of the water molecules when the water molar fraction exceeds 0.10 . In contrast, both the experimental and simulated self-diffusion coefficients of ions were about one-fifth of the water self-diffusivity and were also affected by the water molar fraction.
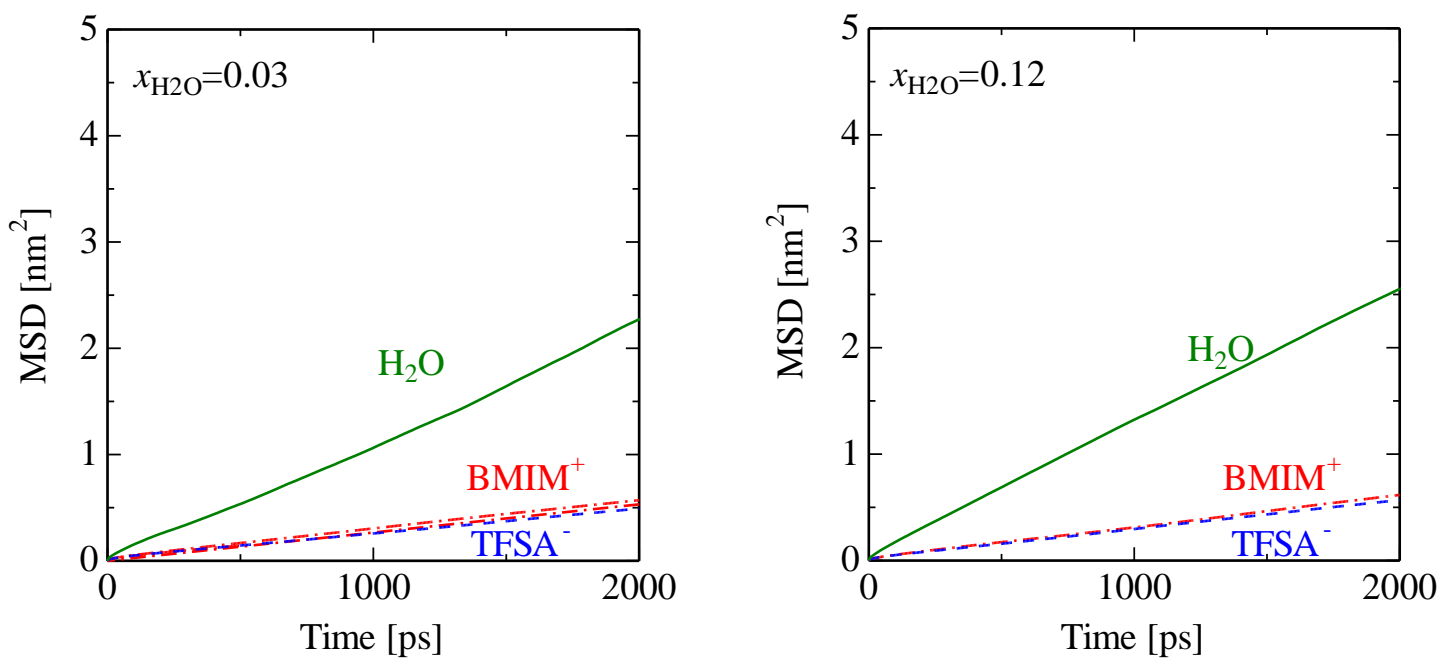

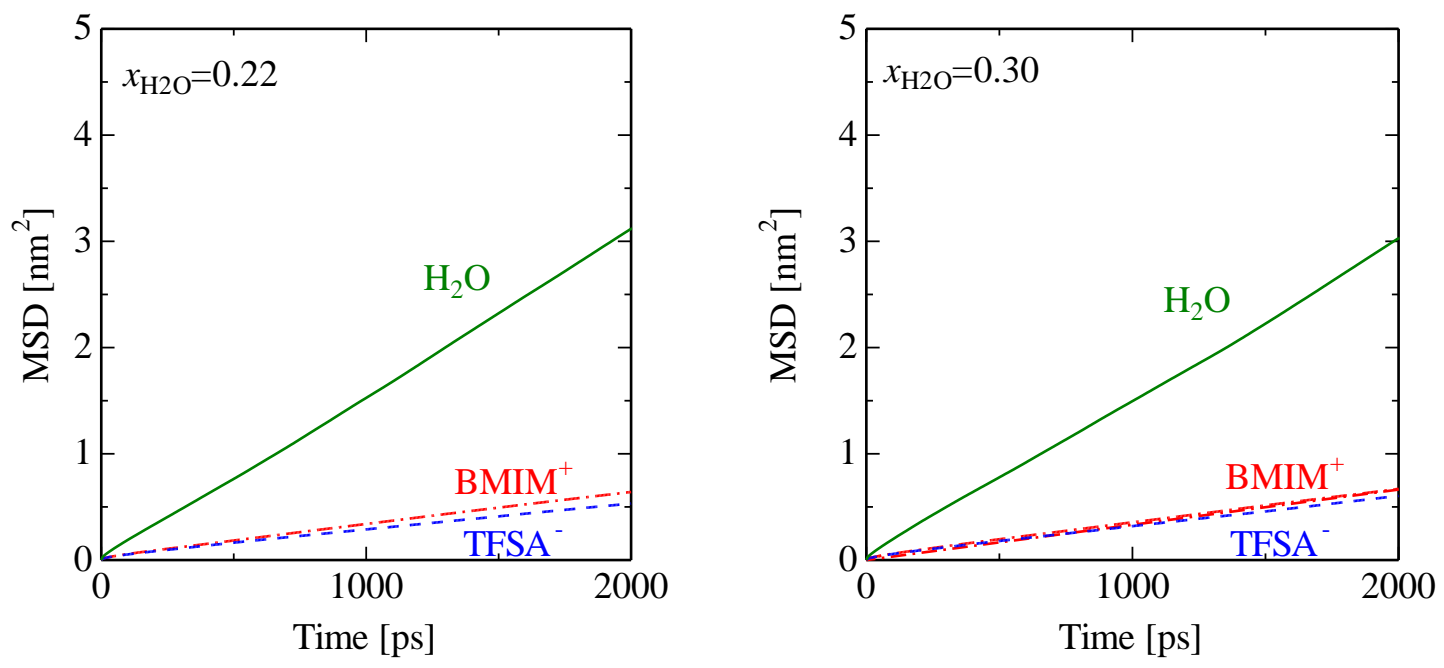

Figure 2 Mean square displacement of cation, anion, and water molecules for the [BMIM][TFSA]/water system

Table 2 Self-diffusion coefficients of $\mathrm{BMIM}^{+}$, TFSA', and water for the [BMIM][TFSA]/water system calculated by molecular dynamics simulation

\begin{tabular}{ccccccc}
\hline & \multicolumn{5}{c}{$D \times 10^{10}\left[\mathrm{~m}^{2} / \mathrm{s}\right]$} \\
\hline $\begin{array}{c}\text { Molar fraction } \\
\text { of water }\end{array}$ & Exp. & Calc. & Exp. & Calc. & Exp. ${ }^{* 2}$ & Calc. \\
\hline 0 & $0.275^{* 1}$ & 0.44 & $0.218^{* 1}$ & 0.39 & & \\
0.03 & $0.230^{* 2}$ & 0.45 & $0.200^{* 2}$ & 0.37 & 0.350 & 1.76 \\
0.12 & $0.260^{* 2}$ & 0.46 & $0.230^{* 2}$ & 0.45 & 1.34 & 2.11 \\
0.22 & $0.270^{* 2}$ & 0.51 & $0.250^{* 2}$ & 0.41 & 2.06 & 2.53 \\
0.30 & $0.290^{* 2}$ & 0.53 & $0.260^{* 2}$ & 0.47 & 2.68 & 2.49 \\
\hline
\end{tabular}

*1 Experimental values were calculated by the equation proposed by Tokuda et al. [1]

*2 Experimental values were cited from Rollet et al. [7] 


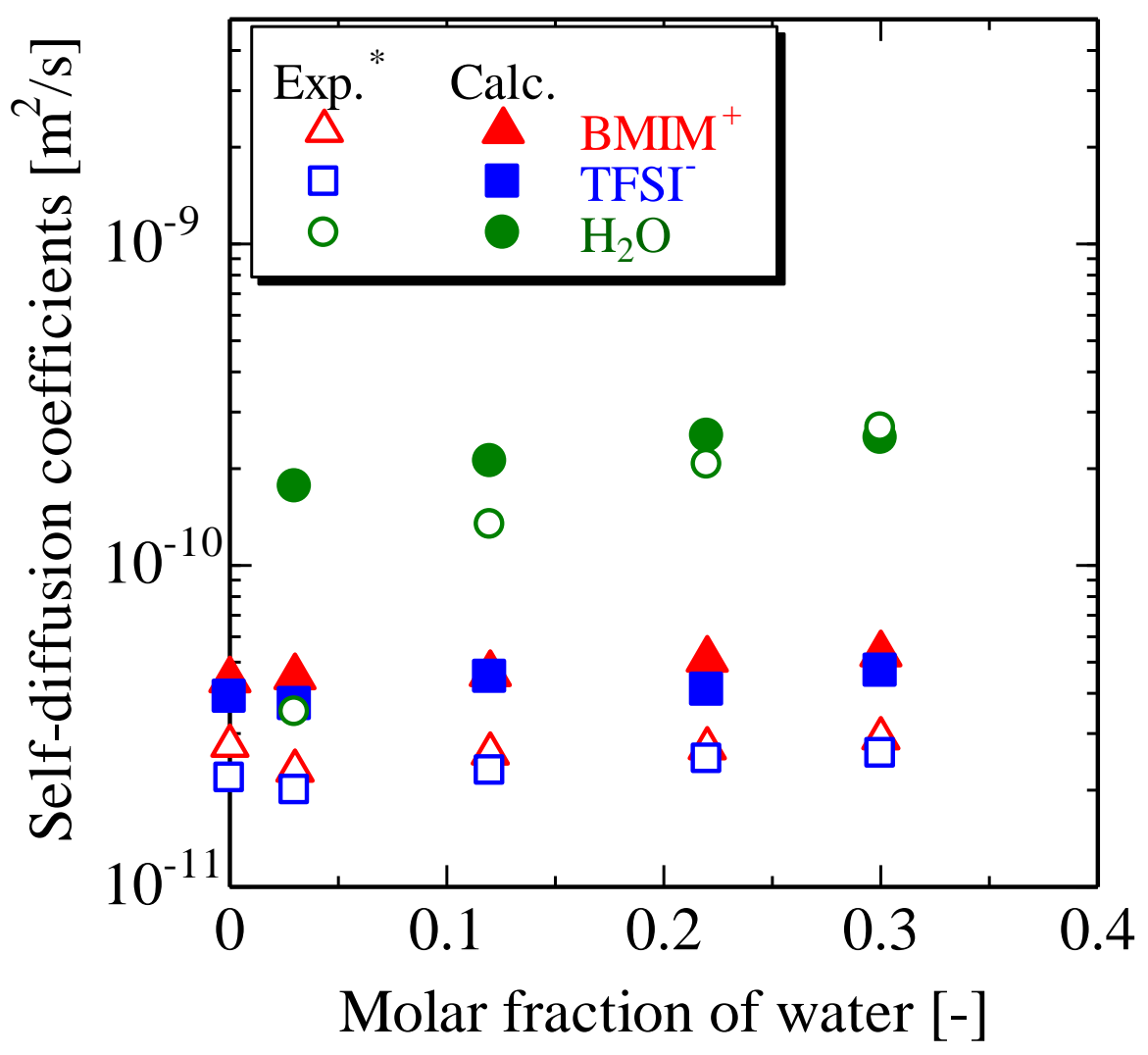

Figure 3 Self-diffusion coefficients of ions and water for the [BMIM][TFSA]/water system (* Experimental data were cited from Tokuda et al. [1] and Rollet et al. [7].)

\subsection{Radial distribution function}

Figure 4 shows the radial distribution functions related to the cation and anion $\left(\mathrm{H}_{2} \mathrm{O}-\mathrm{BMIM}^{+}, \mathrm{H}_{2} \mathrm{O}-\mathrm{TFSA} A^{-}\right.$and $\left.\mathrm{BMIM}^{+}-\mathrm{TFSA}^{-}\right)$. The first peak of the radial distribution functions are relatively small $(g(r)<3)$. The radial distribution

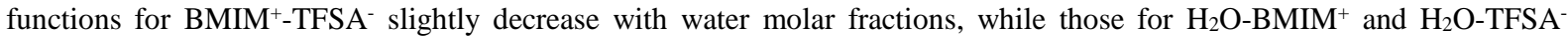
change from one water molar fraction to the next, presumably due to the weak interaction between ions and water and the slight change of the solvation structure of [BMIM][TFSA]/water at different water molar fractions. The slight change of the solvation structure might be one of the main factors underlying the small concentration dependence of the self-diffusion coefficients of the cation and anion. 
Figure 5 (a) plots the radial distribution function for $\mathrm{H}_{2} \mathrm{O}-\mathrm{H}_{2} \mathrm{O}$. As the plot shows, the function was relatively affected by the water molar fractions. The snapshot images of water configuration for $x_{\mathrm{H} 2 \mathrm{O}}=0.12$ and 0.30 are shown in Figures 5 (b) and (c) (the cation and anion were deleted after the simulation). The first highest peaks of the radial distribution function are calculated for $\mathrm{H}_{2} \mathrm{O}-\mathrm{H}_{2} \mathrm{O}$ at the smallest distance (around $2.7 \AA$ corresponding the distance of hydrogen bonding of OH-O, see Fig. 5(a)), and the peak heights are almost the same at any water molar fraction. The peak height of first peak for $\mathrm{H}_{2} \mathrm{O}-\mathrm{H}_{2} \mathrm{O}$ in water-ionic liquid mixture is about 10 times larger than that of pure water. It would indicate that the proportion of water molecules which were neighbor to the other water molecules was so large and the small water clusters consist of a few water molecules would disperse in the ionic liquid at lower water molar fraction $\left(x_{\mathrm{H} 2 \mathrm{O}}<0.12\right.$, see Fig. $\left.5(\mathrm{~b})\right)$. The second peak corresponding to the formation of $\mathrm{H}_{2} \mathrm{O}-\mathrm{H}_{2} \mathrm{O}-\mathrm{H}_{2} \mathrm{O}$ appears at around $5 \AA$, and the peak height gradually increases with the water molar fraction. On the other hand, the third peak appears at around $7.5 \AA$ and the peak heights are almost the same at any water molar fraction. The shape of the radial distribution function for $\mathrm{H}_{2} \mathrm{O}-\mathrm{H}_{2} \mathrm{O}$ in water-ionic liquid mixture at higher water molar fraction will get closer to the function of pure water. The small clusters of water molecules would disperse at lower water molar fraction $\left(x_{\mathrm{H} 2 \mathrm{O}}<0.12\right)$, but the clusters would agglomerate at higher water molar fraction $\left(x_{\mathrm{H} 2 \mathrm{O}}>0.22\right.$, see Fig. 5(c)). The water molecules in the ionic liquid therefore move in the form of aggregated water clusters. The self-diffusion coefficients of pure water is about $2.3 \times 10^{-9} \mathrm{~m}^{2} \mathrm{~s}^{-1}$ and one order larger than that in water-ionic liquid system. Therefore, the self-diffusion coefficients of water in the ionic liquid is enhanced with molar fraction of water by moving water molecules through the water cluster. Rollet et al. [7] described the existence of a similar 'water pool' in the ionic liquid. The snapshot image in Figure 5 (c) depicts water molecules forming clusters of this type. The size of 'water pool' in the ionic liquid was estimated to be 10 to $20 \AA$ (consist of 3 to more than 10 water molecules) by the present simulation. As a future study, the existence of such 'water pool' in ionic liquid and their size may be experimentally validated by using advanced techniques such as X-ray diffraction. From the MD simulation, we presume that the significant enhancement of the self-diffusion coefficients of water as the water molar fraction rose in the experiments originated from the formation of water clusters in the 

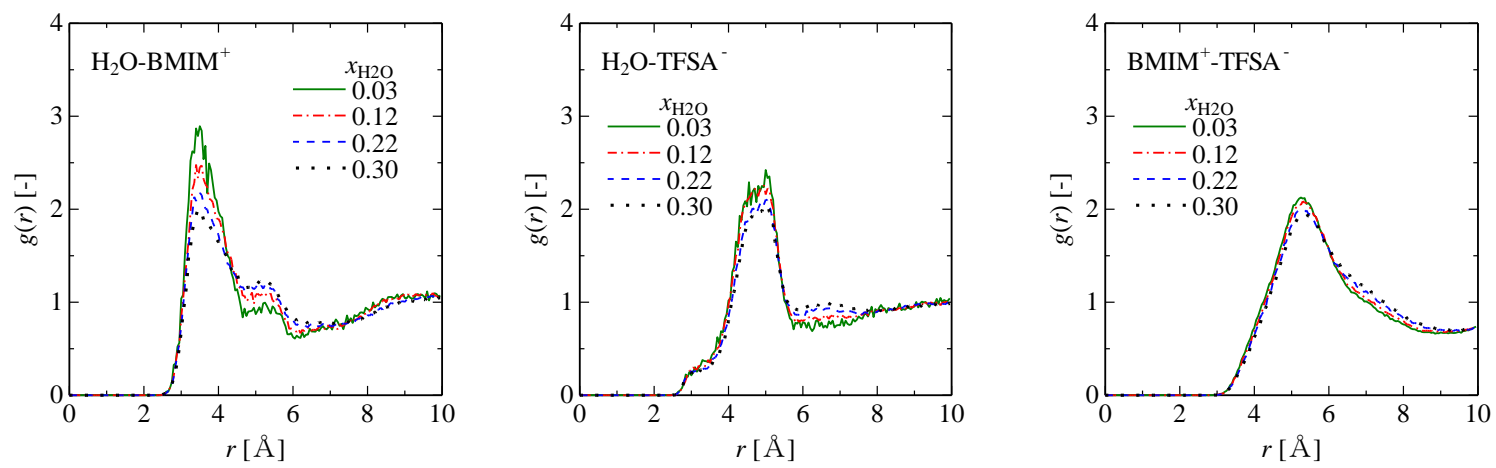

Figure 4 Radial distribution functions for $\mathrm{H}_{2} \mathrm{O}-\mathrm{BMIM}^{+}, \mathrm{H}_{2} \mathrm{O}-\mathrm{TFSA}^{-}$and $\mathrm{BMIM}^{+}-\mathrm{TFSA}^{-}$for the [BMIM][TFSA]/water system

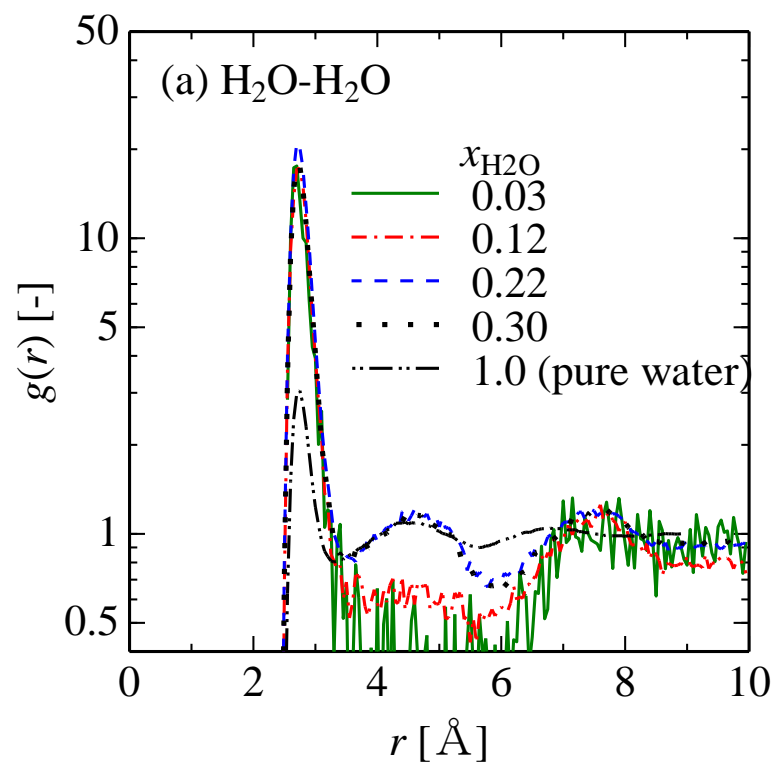

(b)

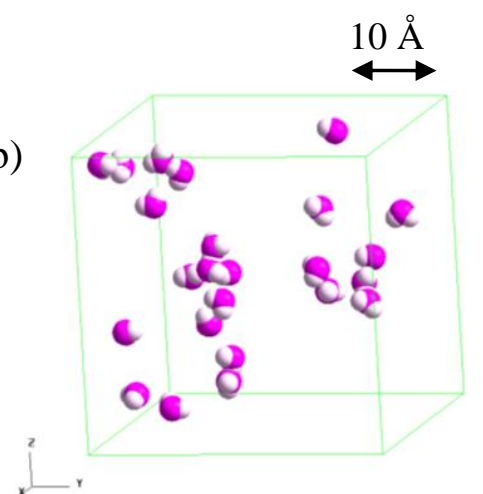

(c)

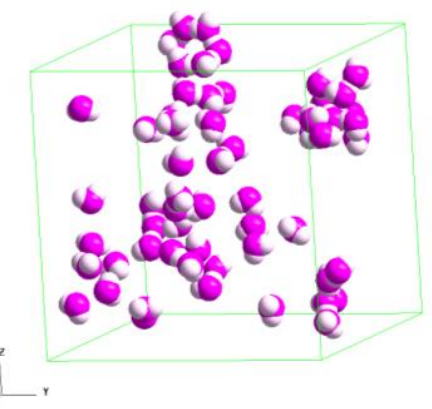

Figure 5 (a) Radial distribution function for $\mathrm{H}_{2} \mathrm{O}-\mathrm{H}_{2} \mathrm{O}$ and water configuration $\left((\mathrm{b})_{\mathrm{H}_{2} \mathrm{O}}=0.12\right.$, (c) $x_{\mathrm{H} 2 \mathrm{O}}=0.30$, except for the cation and anion) for the [BMIM][TFSA]/water system

\section{CONCLUSION}

The self-diffusion coefficients of cation, anion, and water molecules for the [BMIM][TFSA]/water system were 
calculated by molecular dynamics simulation. The experimentally observed enhancement of the self-diffusion coefficients of water with an increasing water concentration was qualitatively reproduced by the simulated results. The formation of aggregated water clusters, what Rollet et al. [7] described as a "water pool," appears to be the source of the anomalous enhancement of the self-diffusion coefficients of water in the ionic liquid by the solution structure indicated in the calculated radial distribution functions.

\section{REFERENCES}

[1] Tokuda, H., K. Hayamizu, K. Ishii, M.A.B.H. Susan and M. Watanabe; Journal of Physical Chemistry B, 108 (2004) $16593-16600$

[2] Tokuda, H., K. Hayamizu, K. Ishii, M.A.B.H. Susan and M. Watanabe; Journal of Physical Chemistry B, 109 (2005) 6103-6110

[3] Tokuda, H., K. Ishii, M.A.B.H. Susan, S. Tsuzuki, K. Hayamizu and M. Watanabe; Journal of Physical Chemistry B, 110 (2006) 2833-2839

[4] Filippov, A., F.U. Shah, F.U., M. Taher, S. Glavatskih and O.N. Antzutkin; Physical Chemistry Chemical Physics, 15 (2013) 9281-9287

[5] Filippov, A., N. Azancheev, M. Taher, F.U. Shah, P. Rabét, S. Glavatskih and O.N. Antzutkin; Magnetic Resonance in Chemistry, 53 (2015) 493-497

[6] Kruk, D., R. Meier, A. Rachocki, A. Korpała, R.K. Singh and E.A. Rössler; Journal of Chemical Physics, 140 (2014) 244509

[7] Rollet, A.-L. P. Porion, M. Vaultier, I. Billard, M. Deschamps, C. Bessada and L. Jouvensal; Journal of Physical Chemistry B, 111 (2007) $11888-11891$

[8] Menjoge, A., J. Dixon, J. F. Brennecke, E. J. Maginn, and S. Vasenkov, Journal of Physical Chemistry B, 113 (2009) 
6353-6359

[9] Kowsari, M.H., S. Alavi, M. Ashrafizaadeh and B. Najafi; Journal of Chemical Physics, 129 (2008) 224508

[10] Kowsari, M.H., S. Alavi, M. Ashrafizaadeh and B. Najafi; Journal of Chemical Physics, 130 (2009) 014703

[11] Jiang, H., F. Zhao, J. Wang, Z. Liu, J. Ren, R. Liu, J. Shang and Y. Hu; Journal of Molecular Liquids, 165 (2012) 63-70

[12] Chen, M., R. Pendrill, G. Widmalm, J.W. Brady and J. Wohlert; Journal of Chemical Theory and Computation, 10 (2014) 4465-4479

[13] Matsumiya, M., K. Hata and K. Tsunashima; Journal of Molecular Liquids, 203 (2015) 125-130

[14] Tsuzuki, S., W. Shinoda, H. Saito, M. Mikami, H. Tokuda and M. Watanabe; Journal of Physical Chemistry B, 113 (2009) 10641-10649

[15] Köddermann, T., D. Paschek and R. Ludwiq; ChemPhysChem, 8 (2007) 2464-2470

[16] Zhong, X., Z. Liu and D. Cao; Journal of Physical Chemistry B, 115 (2011) 10027-10040

[17] Niazi, A. A., B. D. Rabideau and A. E. Ismail; Journal of Physical Chemistry B, 117 (2013) 1378-1388

[18] Ewald, P.P.; Ann. Phys., 64 (1921) 253-287

[19] Berendsen, H. J. C., J. R. Grigera and T. P. Straatsma; Journal of Physical Chemistry, 91 (1987) 6269-6271

[20] Lee, S. H.; Bull. Korean Chem. Soc., 34 (2013) 3800-3804

[21] Nose, S.; Journal of Chemical Physics, 81 (1984) 511-519

[22] Hoover, W. G.; Physical Review A, 31 (1985) 1695-1697

[23] Berendsen, H. J. C., J. P. M. Postma, W. F. Vangunsteren, A. Dinola and J. R. Haak; Journal of Chemical Physics, 81 (1984) 3684-3690

[24] Ivanovskis, G., G. E. Norman and D. R. Usmanova; Doklady Physics, 57 (2012) 427-430

[25] de Andrade, J., H. Böes and E. Stassen; J. Phys. Chem., B, 106 (2002) 13344-13351

[26] Liu, Z., S. Huang and W. Wang; J. Phys. Chem. B, 108 (2004) 12978-12989 
